\title{
Performance Analysis of 4G Systems with Channel Coding Algorithms
}

\author{
${ }^{* 1}$ C. Padmaja, ${ }^{2}$ B.L. Malleswari \\ ${ }^{* 1,2}$ ECE Dept., GNITS, Hyderabad \\ ${ }^{*}$ Email: padmaja.chennapragada@gmail.com
}

Received: 06th October 2017 Accepted: 14th November 2017, Published: 31st December 2017

\begin{abstract}
The performance of wireless communication system is degraded by the burst errors due to deep fades under multipath environment. So, techniques like forward error correction algorithms or automatic repeat request (ARQ) or a combination of both (hybrid ARQ) eliminates the bit error rate (BER). The paper focuses on the performance comparison analysis of different error control codes that gives the near Shannon capacity.
\end{abstract}

Keywords: Shannon Capacity; Bit Error Rate; Forward Error Correction Codes; Signal to Noise Ratio.

\section{INTRODUCTION}

The wireless channel is often affected by atmospheric conditions such as scattering and reflections from surroundings. These multiple signals with different amplitudes and phases reaches the receiver, thus causes either constructive or destructive fading [1]

The poor performance of a reliable communication depends on the received signal strength. The deep fade event due to destructive phenomenon depends on the fading channel coefficient magnitude, 'a'

$$
\text { i.e., } a<\frac{1}{\sqrt{S N R}} \text {. }
$$

Communication receiver will suffer from errors due to deep fades. To combat the deep fading and to improve the performance of the system, employ the technique called "diversity" [2-3].

Diversity is a process of sending the same information symbols over multiple independent identically distributed fading paths, the maximum diversity gain can be achieved. Also, maximizes the output SNR by combining each branch SNR using diversity combiner.

The probability of deep fade event $\left(\mathrm{P}_{\mathrm{DF}}\right)$ decreases as SNR increases. For ' $L$ ' paths between transmitter and receiver, $\mathrm{P}_{\mathrm{DF}}$ decreases as $\left(1 / \mathrm{SNR}^{\mathrm{L}}\right)$ due to multiple fading IID channels employing Diversity [4-5].

Shannon's capacity defines the maximum error-free data transmission rate for a given bandwidth and signal power and received noise power [6]. The channel encoder improves the bit error rate and performance of transmission link over the multipath fading channels [7].

The code rate is defined as the fraction of $k$ information symbols and $n$ encoded symbols denoted by $k / n$. The maximum code rate depends on the type of error correcting code used. The error rate, capacity and throughput can be improved by using multicarrier modulation technique called
Orthogonal Frequency Division Multiplexing OFDM [8] [10].

For an AWGN channel, the theoretical comparison analysis under BPSK modulation between the coded and uncoded system w.r.t. Shannon's capacity is given below.

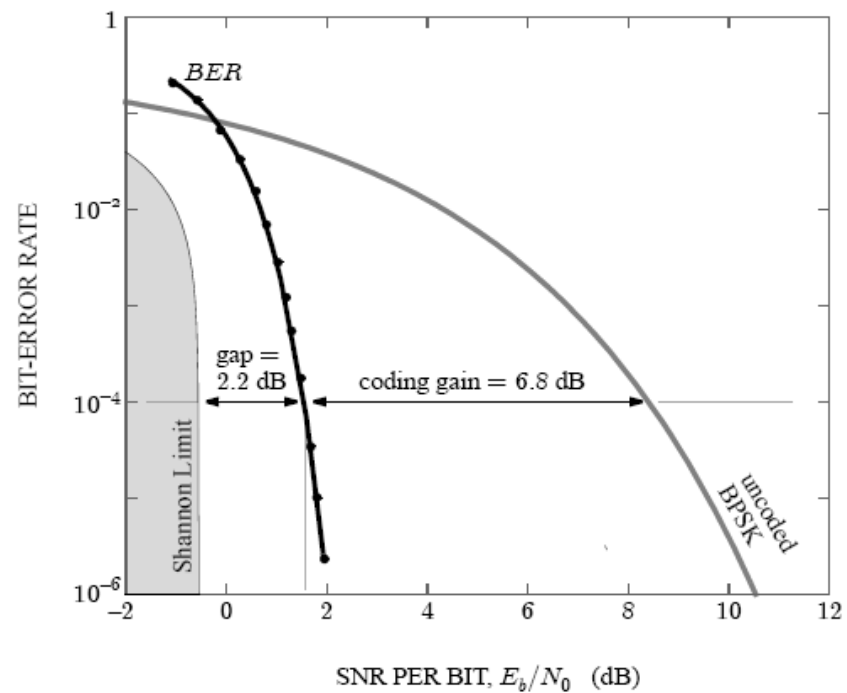

Fig.1.: Theoretical analysis of coding gain [15]

There are number of error correcting codes in the literature such as convolutional codes, block codes, Turbo codes or Low Density Parity Check codes (LDPC) and so on. They differ in terms of decoding complexity, efficiency and optimality [11-13].

The paper evaluates the comparison analysis of convolutional coded system, Turbo coded parallel concatenation convolutional code and uncoded system interms of decoding complexity [14-15].

Section 2 deals with the system model and the analysis of various coding schemes described in section 3 and section 4 lists the simulation results under Rayleigh fading channel. Section 5 concludes the paper.

\section{SYSTEM MODEL}

Consider an OFDM based system employing diversity gain through space encoding technique and channel coding through Turbo coded technique.

The information bits are encoded by Turbo channel encoder and then mapped by digital MQAM modulation. The mapped data is again encoded by Orthogonal Space Time Block Code i.e., OSTBC encoder. The independent data streams are passed through OFDM modulators which perform by IFFT and add Cyclic Prefix (CP) then it is passing through IID channel. 
At the receiver, the received signals are passed through OFDM demodulators where it first discard the $\mathrm{CP}$ and perform an N-point FFT. The OFDM demodulator's outputs are finally separated and passed through OSTBC decoder. This data is demodulated and then Turbo decoded using log MAP algorithm.

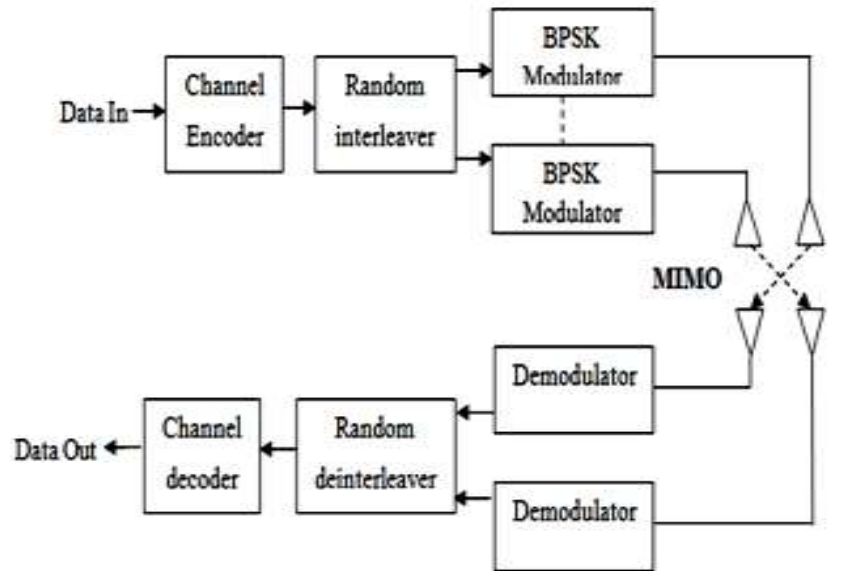

Fig. 3.: Block diagram of proposed system

OFDM is a multicarrier modulation technique used to convert wide band frequency selective channel into parallel narrowband flat fading channels which suppresses the Inter Symbol Interference (ISI). At the receiver, after the FFT stage, the received signal can be written as [2]:

$$
Y(k)=H(k) \cdot X(k)+N(k)
$$

Where,

$\mathrm{N}=$ Number of sub-carriers

$\mathrm{L}=$ Number of channel taps

$\mathrm{K}=\mathrm{K}^{\text {th }}$ sub-carrier

$\mathrm{H}(\mathrm{k})=$ Rayleigh fading coefficient with average power ' $\mathrm{L}$ ' and $\mathrm{N}(0, \mathrm{~L})$

$\mathrm{X}(\mathrm{k})=\mathrm{k}^{\text {th }}$ FFT point of Transmitted symbol with average power ' $\mathrm{P}$ '

$\mathrm{Y}(\mathrm{k})=\mathrm{k}^{\text {th }}$ FFT point of Received symbol

$\mathrm{N}(\mathrm{k})=$ Gaussian noise with $\mathrm{N}\left(0, \mathrm{~N}^{2}\right)$.

Then SNR at the output of FFT can be, $S N R_{r}=\frac{|H(k)|^{2} \cdot P}{N \cdot \sigma^{2}}=L\left(\frac{P}{N \sigma^{2}}\right)$

$\therefore B E R=Q\left(\sqrt{S N R_{r}}\right)$

$\Rightarrow B E R=\frac{1}{2}\left(1-\sqrt{\frac{S N R_{r}}{2+S N R_{r}}}\right)$

$\Rightarrow B E R=\frac{1}{2}\left(1-\sqrt{\frac{(L / N) S N R}{2+(L / N) S N R}}\right)$

(3)

Let $h 1$ and $h 2$ are the complex fading coefficients on the 1 st and the 2 nd transmit antennas, being Rayleigh distributed and $n 1$ and $n 2$ are complex Gaussian random variables of zero mean and variance $\sigma^{2}$. Assuming perfect knowledge of the fading coefficients at the receiver, then the probability of error for the bit will be a function of $h 1$ and $h 2$.

$$
\begin{aligned}
& P_{e}=Q\left(\sqrt{S N R_{m}}\right)=Q\left(\sqrt{\left(\left\|h_{1}\right\|^{2}+\left\|h_{2}\right\|^{2}\right) S N R}\right) \\
& \therefore \text { AverageBER }=\frac{1}{2}\left(1-\lambda_{1}\right)^{2}\left(2+\lambda_{1}\right)+\frac{1}{4}\left(1-\lambda_{2}\right)^{2}\left(2+\lambda_{2}\right)+\frac{1}{4}\left(1-\lambda_{3}\right)^{2}\left(2+\lambda_{3}\right) \\
& \text { where } \\
& \lambda_{1}=\sqrt{\frac{S N R}{5+S N R}} ; \lambda_{2}=\sqrt{\frac{9 S N R}{5+9 S N R}} ; \lambda_{1}=\sqrt{\frac{25 S N R}{5+25 S N R}} ;
\end{aligned}
$$

\section{Performance Analysis of Turbo coded OSTBC SYSTEM}

\section{A. Convolutional Code:}

The convolutional code converts the entire input stream into one single code-word. The encoded bit depends not only on the current bit but also on the previous bit information. The decoding is traditionally done using the Viterbi algorithm.

Low rate codes such as convolutional codes having more redundant bits can usually correct more errors. That means that the communication system can operate at lower transmit power, tolerate more interference and noise and transmit at higher data rate. Thus the code becomes more energy efficient.

\section{B. Convolutional Turbo codes:}

The first turbo code, based on convolutional encoding, was introduced in 1993 where a scheme was described that uses a rate $1 / 2$ code over an AWGN channel and achieves a bit error probability of $10^{-5}$ using Binary Phase Shift Keying (BPSK) modulation at SNR of $0.7 \mathrm{~dB}$.

In recent times, turbo codes are finding use in $4 \mathrm{G}$ mobile communications. The concatenation of error correcting codes to raise the near Shannon capacity is known as turbo code.

\section{Turbo Encoder:}

The fundamental turbo code encoder as shown in figure is built using two identical recursive systematic convolutional (RSC) encoders concatenated in parallel.

In turbo code encoder, both the RSC encoders are of short constraint length in order to avoid excessive decoding complexity.

An RSC encoder is typically of rate $r=1 / 2$ and is termed a component encoder. The two component encoders are separated by an interleaver.

The output of the turbo encoder consists of the systematic input data and the parity outputs from two constituent RSC encoders. The systematic outputs from the two RSC encoders are not needed because they are identical to each other (although ordered differently) and to the turbo code input. Thus the overall code rate becomes $r=1 / 3$. 
Helix Vol. 8(1): 2742- 2746

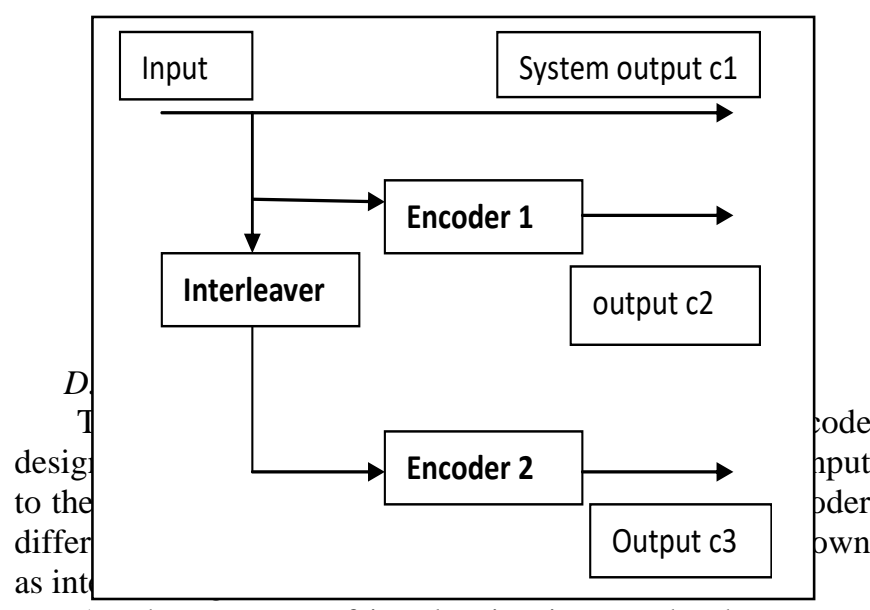

Another purpose of interleaving is to make the outputs of the two encoders uncorrelated from each other. Thus, the exchange of information between the two decoders while decoding yields more reliability.

There are different types of interleavers, e.g., row column interleaver, helical interleaver, odd-even interleaver, etc.

\section{E. Turbo Decoder:}

The turbo decoder consists of two MAP decoders that are interleaved to exchange soft information iteratively until a hard decision is made on the transmitted symbol. These decoders are serially concatenated via an interleaver as shown in Figure.

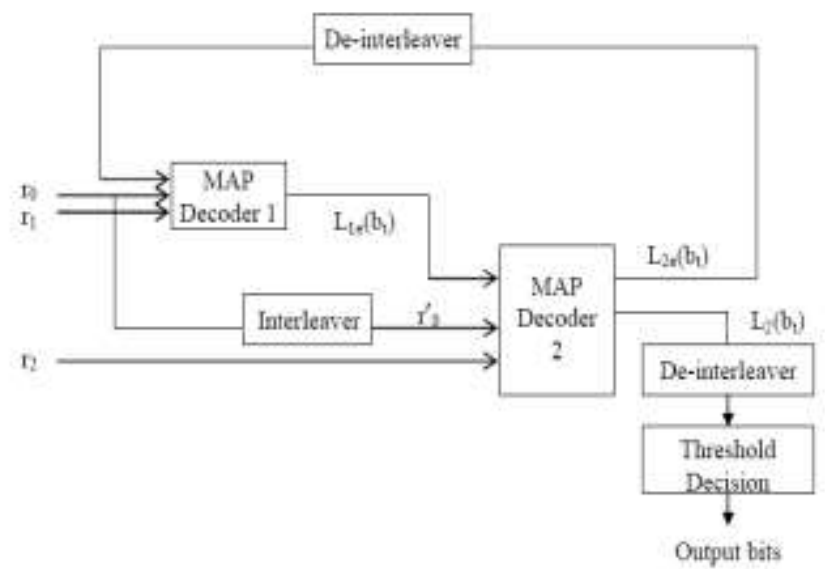

Fig.5.: Turbo Decoder using log MAP

The use of iterative decoding using log-MAP algorithm results in the good performance of turbo codes. In log-MAP decoding, soft decision values are formed using loglikelihood ratios (LLRs) and are based on minimizing the bit error probability, given a transmitted sequence.

The outputs represented by $\mathrm{L}_{1 \mathrm{e}}(\mathrm{bt})$ and $\mathrm{L}_{2 \mathrm{e}}(\mathrm{bt})$ refer to the extrinsic information of the input bit $b_{t}$, defined as the information obtained at the output of each decoder, considering only the parity bits of the encoder. Part of the encoded sequence refers to the original message and is decoded by the first decoder and the second part refers to the interleaved version of the original message and is decoded by the second decoder.
At the beginning of the decoding process, the apriori probability of the original bits are equal and given by

$$
\operatorname{Pr}(1)=\operatorname{Pr}(0)=1 / 2
$$

These apriori probabilities are improved by the two decoders during the iteration process and soft output estimates or LLRs are obtained. The symbols to be decoded are passed through decoder 1 and the outputs are interleaved and the extrinsic information thus extracted is then passed through decoder 2.

These estimates are mutually fed back and are interleaved by the same interleaving pattern that was used at the transmitter. By using MAP algorithm, the second decoder obtains the LLR $\mathrm{L}_{2}\left(\mathrm{~b}_{\mathrm{t}}\right)$ as:

$$
b=\left\{\begin{array}{l}
1, L_{2}\left(b_{t}\right) \geq 0 \\
0, \text { otherwise }
\end{array}\right\}
$$

The likelihood function of $\mathrm{y}_{\mathrm{i}}$ will be,

$$
p\left(y_{i} \mid x_{i}\right)=\frac{1}{\sqrt{2 \Pi \sigma^{2}}} \exp \left(-\frac{\left(y_{i}-\mu\right)^{2}}{2 \sigma^{2}}\right)
$$

Then the log likelihood ratio of the channel will be,

$L L R=\log \left(\frac{p\left(y_{i} \mid x_{i}=1\right)}{p\left(y_{i} \mid x_{i}=0\right)}\right)$

Thus the LLR equation can be broken into three terms as

$\mathrm{L}\left(\mathrm{b}_{\mathrm{t}}\right)=$ Lapriori+Lchannel+Lextrinsic

(9)

where it is evident that $L\left(b_{t}\right)$ consists of three elements, being that $\mathrm{L}_{\text {channel }}$ reflects the channel's pdf, $L_{\text {apiori }}$ the a priori information, and $L_{\text {extrinsic }}$ and the derived probability of the decoder itself.

\section{Simulation Parametrers}

Performance results for the Turbo Concatenated Hybrid Space Time Code are provided in this section.Let the

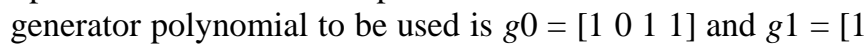
$\left.\begin{array}{lll}0 & 1\end{array}\right]$. Rayleigh fading assumption is made and the noise is Gaussian with zero mean and variance N0. It is also assumed that perfect CSI is available at the receiver. The path gains are constant for a period of 40 transmissions. For a block length of 40 symbols, the frame size for 10 blocks is 400 symbols and the interleaver size is 80 symbols. The spectral efficiency of the concatenated system is $2 \mathrm{bps} / \mathrm{Hz}$. 
Helix Vol. 8(1): 2742- 2746

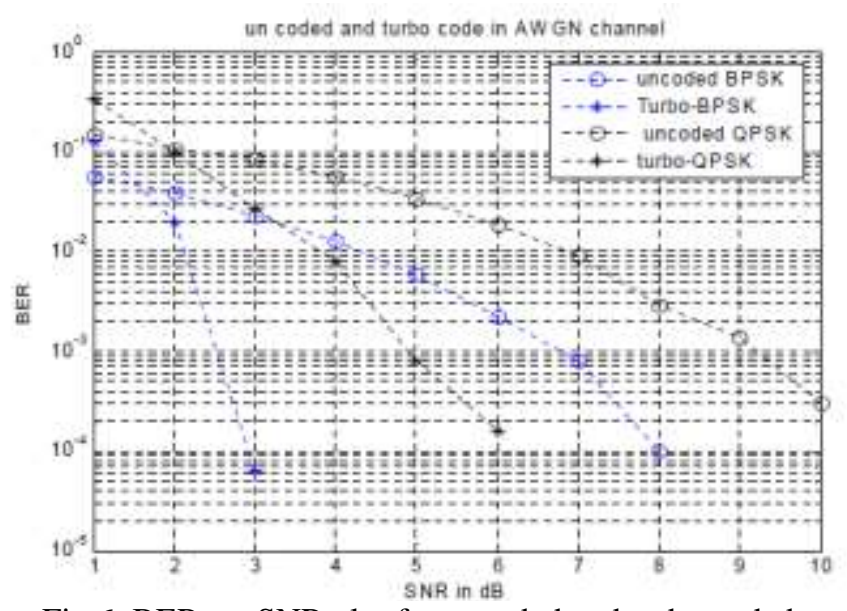

Fig 6: BER vs. SNR plot for uncoded and turbo coded OFDM using BPSK and QPSK.

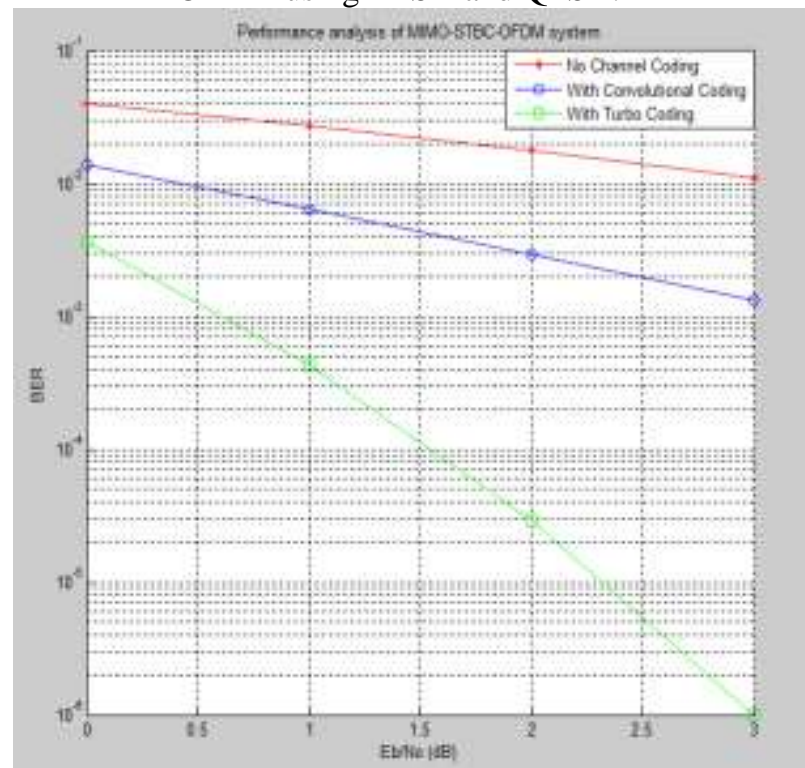

Fig 7: Performance comparison analysis of turbo coded MIMO-STBC-OFDM system.

In figure 6 and 7 , without channel coding the system reached a BER of about $10^{-2}$ at $3 \mathrm{~dB}$, with convolutional coding reached a BER of about $10^{-3}$ at $3 \mathrm{~dB}$ and using turbo coding which gave a simulated BER limit of $10^{-6}$ at same $3 \mathrm{~dB}$. From simulation results the turbo code gives the best BER performance at low SNR levels thereby enhancing reliability in high speed data transmission over multipath fading channels.

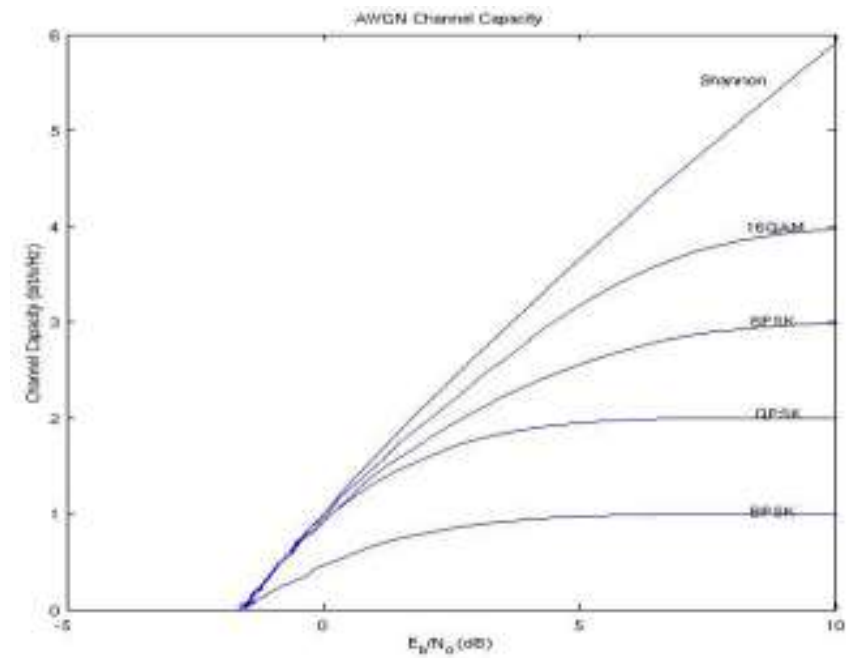

Fig.8 :Spectral efficiency verses SNR

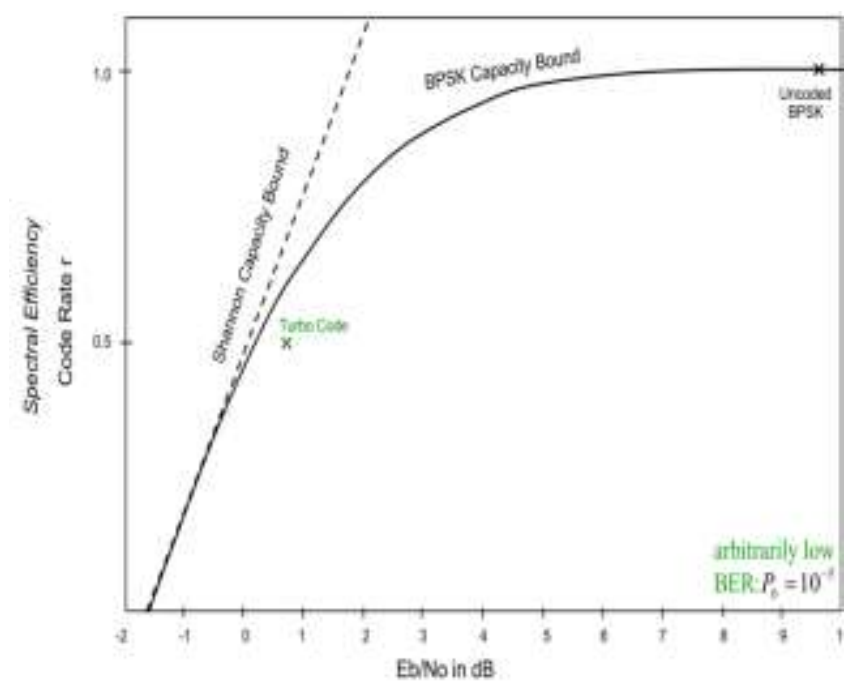

Fig.9 : Turbo code performance approaches Shannon capacity

From figure 8 , the spectral efficiencies of different modulation schemes w.r.t. Shannon's capacity is analyzed. It is clear that as the modulation order increases, the system approaches the Shannon's limit. From figure 9, recursive turbo decoding achieve near Shannon's limit performance in the turbo cliff region.

\section{Conclusions}

Implementation using turbo coded MIMO-STBCOFDM system results in better reliability for high speed data transmission over multipath Rayleigh fading channels as compared to uncoded and Convolutional coded MIMOSTBC-OFDM. Also, convolutional decoding using Viterbi algorithm works by rejecting least likely paths at early stages of decoding process yields loss of information. The turbo decoding relies on passing bit by bit soft information about individual transmitted bits from one decoding stage to the next. Further improvement in the performance can be obtained by applying turbo code which gives better performance at low SNR. 


\section{References}

[1] SALTBURG B. R. (1967), Performance of efficient parallel data transmission systems, IEEE Trans. on Comm. Tech., pp. 805-811.

[2] Weinstein S.B. and P. M. Ebert, Data transmission by frequency-division multiplexing using the discrete Fourier transform, IEEE Trans. Commun. Technol., vol. COM-19, pp. 628-6343, Oct. 1971.

[3] A. Peled and A. Ruiz, "Frequency Domain Data Transmission using Reduced Computational Complexity Algorithms," Acoustics, Speech, and Signal Processing, IEEE International Conference on ICASSP '80, Vol. 5, pp.964 - 967, Apr. 1980.

[4] W.E. Keasler, Jr., "Reliable Data Communications over the voice band-width Telephone Using Orthogonal Frequency Division Multiplexing," Ph.D. dissertation, Univ. Illinois, Urbana, Il, 1982.

[5] B. Hirosaki, "An Analysis of Automatic Equalizers for Orthogonally Multiplexed QAM Systems," IEEE Transaction Communication, Vol.28, pp.73-83, Jan. 1980.

[6] B. Hirosaki, S.Hasegawa, and A. Sabato, "Advanced Group-band Data Modem Using Orthogonally Multiplexed QAM Technique," IEEE Trans. Commun. Vol. 34, no. 6, pp. 587-592, Jun. 1986.

[7] L. J. Cimini, "Analysis and Simulation of a Digital Mobile Channel using Orthogonal Frequency Division multiplexing," IEEE Transaction Communications, Vol.33, pp. 665-675. July 1985.

[8] O. Edfors, M. Sandell, J.-J. van de Beek, S. K. Wilson, and P. O. Börjesson, "OFDM channel estimation by singular value decomposition," IEEE Trans. Commun., vol. 46, pp. 931-939, July 1998.

[9] Y. Li and N. Sollenberger, "Interference suppression in OFDM systems using adaptive antenna arrays," in 1998 IEEE Global Telecomm. Conf.: Commun. The MiniConf., Sydney, Australia, Nov. 1998, pp. 213-218.

[10] Y. Li, N. Seshadri, and S. Ariyavisitakul, "Transmitter diversity of OFDM systems with dispersive fading channels," in 1998 IEEE Global Telecomm. Conf., Sydney, Australia, Nov. 1998, pp. 968-973.

[11] J. K. Cavers, "An analysis of pilot symbol assisted modulation for Rayleigh fading channels," IEEE Trans. Veh. Technol., vol. 40, pp. 686-693, Nov. 1991.

[12]F. Wan, W.-P. Zhu, M.N.S. Swamy, Semiblind sparse channel estimation for MIMO-OFDM systems, IEEE Transactions on Vehicular Technology 60 (July (6)) (2011) 2569-2582.

[13]P. K. Vitthaladevuni and M.-S. Alouini,"BER computation of 4/MQAM hierarchical constellations," IEEE Trans. Broadcasting, vol. 47, no. 3,pp. 228240,September 2001.

[14] K. Cho and D. Yoon, "On the general BER expression of one and two dimensional amplitude modulations," IEEE Trans. Commun., vol. 50, no. 7,pp. 1074-1080,July 2002.

[15] C. Berrou, A. Glavieux, and P. Thitimajshima, "Near Shannon Limit Error-Correcting Coding: Turbo Codes", Proceedings of the IEEE International Conference on Communications ICC '93, Geneva. pp. 1064-1070, May 1993. 